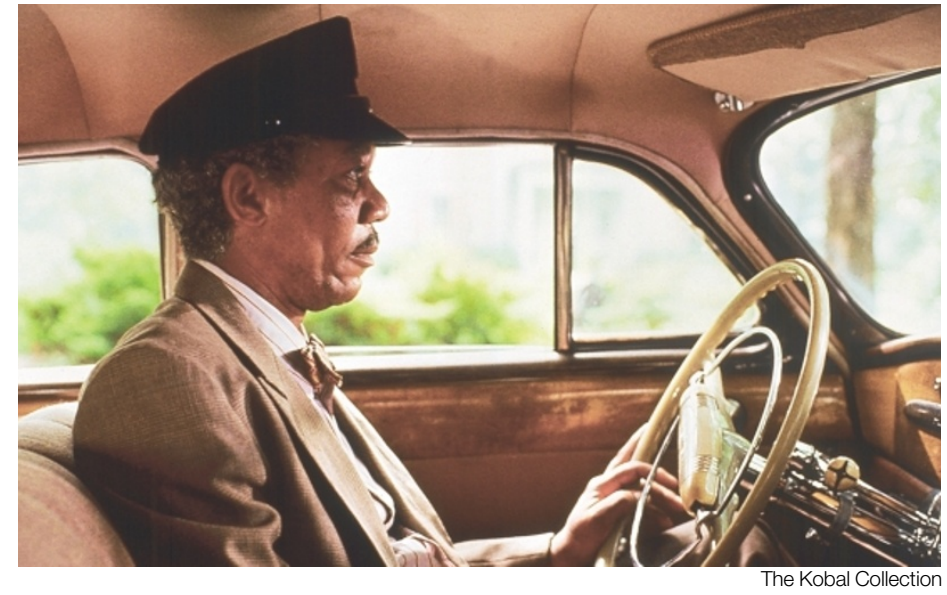

CYTOSKELETON

\section{Molecular chauffeurs}

Nerves rely on microtubule-dependent transport along the axon to ensure that vesicles containing synaptic proteins are delivered to the synapse. Their vehicle - the microtubule-binding motor kinesin - is well characterized. However, the identity of the molecule that mediates the interaction between kinesin and its cargo in axons has remained elusive. Reporting in Cell and Neuron, Lawrence Goldstein and colleagues now show that there might be more than one chauffeur for the job.

To screen for proteins that might mediate this interaction, Bowman et al. used the genetically amenable Drosophila melanogaster. They mutagenized flies and screened larvae for behavioural phenotypes previously characterized in mutants lacking subunits of kinesin-1. Through this, they identified a candidate Sunday driver (SYD).

Their suspicions were confirmed when they found large accumulations of axonal membrane-bound cargos in the nerves of the larvae, indicating problems in axonal transport. The syd gene product contains two predicted protein-protein interaction domains. BLAST analysis showed syd homologues in the worm, mouse and humans. So might SYD be the longsought factor on vesicles that interacts with kinesin-1?

To test this, the authors asked if syd is expressed in the right places. Expression of syd fused to green fluorescent protein in epithelial cells shows that it localizes to the Golgi, and to vesicles of the secretory pathway together with kinesin-1. Furthermore, expression analysis on western blots of mouse brain and extracts from nervous tissue showed that SYD is proba-

bly present in axons.

Next, Bowman et al. tested whether SYD can bind to kinesin-1. They confirmed this using the yeast two-hybrid system and co-immunoprecipitation. To show that this interaction is direct, they checked that the recombinant purified proteins bound together in a GST-pulldown assay. And they narrowed down the region responsible for this interaction to the tetratricopeptide (TPR) domain of the kinesin-1 light chain (KLC).

Does SYD mediate binding of all cargos with kinesin-1? Kamal et al. suggest not. They show that at least one cargo protein — the $\beta$-amyloid precursor protein (APP) - interacts with KLC directly. Intriguingly, APP binds to the same region as SYD. So one possibility is that different cargos compete for interaction with KLC, ensuring their safe delivery to the synapse.

But is it important for SYD to reach the synapse? Two other labs have also recently shown that the mouse syd gene product acts as a scaffold for the mitogen-activated protein kinase cascade, indicating that once SYD has reached the synapse, it might have other jobs beyond chauffeuring.
Alison Schuldt (2) References and links ORIGINAL RESEARCH PAPERS Bowman, A. B. et al. Kinesin-dependent axonal transport is mediated by the Sunday driver (SYD) protein. Ce 103, 583-594 (2000) | Kamal, A. et al. Axonal transport of amyloid precursor protein is mediated by direct binding to the kinesin light chain subunit of kinesin-1. Neuron 28, 449-459 (2000) FURTHER READING $\mathrm{lto}$, $M$. et al. JSAP1, a novel Jun $\mathrm{N}$-terminal protein kinase (JNK)-binding protein that functions as a scaffold factor in the JNK signalling pathway. Mol. Cell. Biol. 19 , 7539-7548 (1999) | Kelkar, N. et al. Interaction of a mitogen-activated protein kinase signalling module with the neuronal protein JIP3. Mol. Cell. Biol. 20, 1030-1043 (2000)

\section{IN BRIEF}

\section{CELL CYCLE}

Targeted disruption of the three Rb-related genes leads to loss of G1 control and immortalization.

Sage, J. et al. Genes Dev. 14, 3037-3050 (2000)

Retinoblastoma and its close relatives p130 and p107 have overlapping functions in controlling the cell cycle. To investigate the functions of each protein in vivo, the authors generated single, double and triple-knockout mouse embryonic fibroblasts. The triple knockouts have a shortened cell cycle, show characteristics of transformed cells, and do not undergo G1 arrest in response to several factors. These results, say the authors, "further the link between loss of cell cycle control and tumorigenesis".

\section{DEVELOPMENT}

\section{DNA methylation in Drosophila melanogaster.}

Lyko, F., Ramsahoyer, B. H. \& Jaenisch, R. Nature 408, 538-539 (2000)

Inactive regions of many eukaryotic genomes contain methylated cytosine residues, but the fruitfly was thought to be an exception until recently. Jaenisch and colleagues now reveal that this methylation is restricted to the early stages of embryonic development. The authors show that methylation decreases during later stages of development, and they predict that this could be due to reduced expression of the enzyme responsible, DNA methyltransferase.

\section{PRIONS}

\section{Binding of disease-associated prion protein to} plasminogen.

Fischer, M. B. et al. Nature 408, 479-483 (2000)

$\mathrm{PrP}^{\mathrm{Sc}}$ is associated with transmissible spongiform encephalopathies, but deposition of this protein alone is not sufficient to damage the brain, indicating that it interacts with other cellular factors to cause disease. Fischer $e t$ al. now show that blood plasminogen binds to $\mathrm{PrP}^{\mathrm{Sc}}$ but not to normal $\mathrm{PrP}^{\mathrm{C}}$. Plasminogen is therefore the first molecule that can reliably discriminate between normal and pathological prion proteins, making it a useful tool for diagnostic purposes.

\section{CELL SIGNALLING}

InsP facilitates store-operated calcium influx by inhibition of $\operatorname{lnsP}_{3}$ 5-phosphatase.

Hermosura, M. C. et al. Nature 408, 735-740 (2000)

Is inositol-1,3,4,5-tetrakisphosphate $\left(\operatorname{Ins}(1,3,4,5) \mathrm{P}_{4}\right)$, like inositol-1,4,5-trisphosphate $\left(\operatorname{Ins}(1,4,5) \mathrm{P}_{3}\right)$, a calcium-releasing second messenger? This study reveals that low $\operatorname{Ins}(1,3,4,5) \mathrm{P}_{4}$ concentrations collaborate with $\operatorname{Ins}(1,4,5) \mathrm{P}_{3}$ by inhibiting the enzyme that inactivates $\operatorname{Ins}(1,4,5) \mathrm{P}_{3}$, whereas higher concentrations have the opposite effect by blocking the Ins $(1,4,5) \mathrm{P}_{3}$ receptor. By sacrificing a proportion of the Ins $(1,4,5) \mathrm{P}_{3}$ pool to make Ins $(1,3,4,5) \mathrm{P}_{4}$, cells can turn calcium signals up or down, according to their needs. 